\title{
Mathematical model of membrane potential formation at $E$. coli growth on nitrite
}

\author{
N.A. Ree*, V.A. Likhoshvai, T.M. Khlebodarova \\ Institute of Cytology and Genetics SB RAS, Novosibirsk, Russia \\ *e-mail:kashev@bionet.nsc.ru
}

Key words: nitrite respiration, membrane potential, mathematical model

Motivation and Aim: The main component of the respiratory chain, leading to ATP synthesis, in Escherichia coli cells, cultivated in anaerobiosis on nitrite, is periplasmic Nrf nitrite reductase. It was shown previously [1], that at low extracellular nitrite concentration, genetic component is not sufficient to describe the experimentally measured dynamic of substrate utilization by $E$. coli cells, in the chemostat. It was assumed that additional mechanism, regulating Nrf activity, is influence of membrane potential on enzyme subunits transport to the periplasm and on formation of active $\mathrm{Nrf}$ enzyme. This work is dedicated to verification and justification of this hypothesis.

Methods and Algorithms: Generalized Hill functions were used to describe expression of operons ( $f d h F, h y c, h y b, h y f, n r f$ и $n i r)$, which are involved in nitrite electron transport chain and nitrite metabolism in E. coli cells. Rate of reactions were described by Michaelis-Menten equations. Parameters of the model were evaluated from the published data or were estimated during model's adaptation to experimental data.

Results: The mathematical model of $E$. coli cells respiration on nitrite at anaerobic conditions was created. The model takes into account molecular-genetic mechanisms of respiratory chain formation, nitrite metabolism regulation and also the kinetic of substrate utilization in stationary growth conditions of the chemostat. Lack of particular data on the mechanism of membrane potential formation, the model describes two hypothetical pathways: with involvement of formatelyase complexes FHL-1 or FHL-2. According to the first pathway, FHL-1 complex, composed of FDH-H formate dehydrogenase and Hyd-3 hydrogenase, oxidizes formate to hydrogen, which diffuse to the periplasm, where is utilized by Hyd-2 hydrogenase, transferring electrons through quinone pool on Nrf reductase. According to the second pathway, FHL-2 complex, composed of FDH-H formate dehydrogenase and Hyd-4 hydrogenase, directly transports protons from the cytoplasm to the periplasm and transfers electrons, generated by oxidation of formate, through quinones on Nrf reductase. The model analysis revealed contribution of membrane potential in resulting nitrite utilizing Nrf activity was about $50 \%$ in the micromolar range on substrate concentration, although adequacy of nitrite dynamic reproduction is independent on the pathway of potential formation.

Conclusion: Membrane potential, independent on the way of its formation, is a necessary mechanism, complementing genetic regulation of periplasmic Nrf activity at nitrite concentrations less, then $1 \mathrm{mM}$.

Acknowledgements: The work was partially supported by the RFBR (No. 16-01-00237a) and Project Fundamental Research of SB RAS (No. 0324-2018-0017).

\section{References}

1. Ree N.A. et al. (2015) About mechanisms of nitrite utilization by Escherichia coli cells under stationary growth cultivation. Math. Biol. Bioinf. 10:193-205. 